\title{
Cardiac rehabilitation in patients with pacemakers and implantable cardioverter defibrillators
}

\author{
Marie Christine Iliou, Jean Christophe Blanchard, Aurélia Lamar-Tanguy, \\ Pascal Cristofini, François Ledru \\ Department of Cardiac Rehabilitation and Secondary Prevention, Corentin Celton Hospital, \\ Assistance Publique Hôpitaux de Paris, Hôpitaux Universitaires Paris Ouest, Issy-les-Moulineaux, France
}

\begin{abstract}
Abbreviations
PM: pacemaker

CRT: cardiac resynchronization therapy

ICD: implantable cardiac defibrillator

AV: atrio-ventricular

LVEF: left ventricular ejection fraction

ECG: electrocardiogram
\end{abstract}

\begin{abstract}
Large subsets of patients admitted in cardiac rehabilitation centers are having a pacemaker, cardiac resynchronization (CRT) or implantable cardiac defibrillator (ICD). Cardiac rehabilitation in patients with implanted electronic devices such as pacemakers or ICD is a unique opportunity not only to optimize the medical treatment, to increase their exercise capacity and improves their clinical condition, but also to supervise the correct functioning of the device. CRT is known to reduce clinical symptoms and slightly increase exercise capacity. But in these patients, the clinical improvements are likely to be explained by both the enhancement of cardiac function induced by the device and by the improved peripheral (muscular and vascular) and cardiac effects of exercise. The additional expected gain by exercise in this population is between 14 to $25 \%$. In patients implanted with an ICD, exercise training
\end{abstract}

Corresponding author: Marie Christine Iliou, Service de Réadaptation Cardiaque et Prévention Secondaire, AP-HP Hôpital Corentin Celton, 4, parvis Corentin-Celton BP 66, 92130 Issy-les-Moulineaux, France.

Tel. +33.1.58004260 - Fax +33.1.58004913.

E-mail: marie-christine.iliou@aphp.fr

Key words: Cardiac rehabilitation; exercise; pacemaker; resynchronization; cardiac defibrillator.

Received for publication: 19 April 2016

Accepted for publication: 30 June 2016

(C) Copyright M.C. Iliou et al., 2016

Tipografia PI-ME Editrice, Italy

Monaldi Archives for Chest Disease Cardiac Series 2016; 86:756

doi: 10.4081/monaldi.2016.756

This article is distributed under the terms of the Creative Commons Attribution Noncommercial License (by-nc 4.0) which permits any noncommercial use, distribution, and reproduction in any medium, provided the original author(s) and source are credited. is safe, without increasing recurrence and need of shocks or anti-tachycardia pacing therapy. A comprehensive cardiac rehabilitation combining exercise training and a psycho-educational intervention improves exercise capacity, quality of life, general and mental health. However, further large scale studies are needed to evaluate the most appropriate management and definitively demonstrate the additional benefit of cardiac rehabilitation in this particular group of patients.

\section{Introduction}

The beneficial effects of cardiac rehabilitation on secondary prevention, as well as psychosocial and physiological health condition are widely established for cardiac patients. International guidelines [1,2] are now available for exercise training and rehabilitation in cardiac patients. Nevertheless, very few of these recommendations include specific sections for patients implanted with conventional pacemakers (PM), cardiac resynchronization devices (CRT) or implanted cardiac defibrillators (ICD) [3].

The accepted indications of cardiac rehabilitation include coronary patients (after an acute coronary syndrome, after any coronary revascularization, in stable coronary artery disease, or refractory angina), heart failure patients, post-cardiac surgery, and high cardiovascular risk patients. Many of those patients may be implanted with a cardiac device.

In 2011, there were 938 PM, 140 CRT and 149 ICD implanted per million inhabitants in Europe [4]. The rate of CRT and ICD implantations is currently rapidly growing in patients with heart failure and reaches now 7 to $17 \%$ and 15 to $19 \%$ respectively $[5,6]$.

Consequently, a large subset of patients admitted in cardiac rehabilitation centers are having an electrophysiological device, and require special attention.

\section{Exercise and conventional pacemakers, CRT and ICD}

Pacemakers and cardiac defibrillators were introduced as life-saving devices. Soon, new functions appeared necessary, such as the increase of heart rate during exercise (rate responsiveness), the adaptation of the atrioventricular $(\mathrm{AV})$ delay to improve left ventricular filling, detection of arrhythmias, etc. The technology has consequently rapidly improved allowing a better and more physiologic adaptation to exercise. However, the complexity of settings and clinical conditions requires specific knowledge.

Stimulation therapy is aimed at reducing symptoms, improving quality of life, functional living and survival. To reach these goals, the ideal device must provide an heart rate acceleration during exercise, while keeping a synchrony between atrial and ventricular contractions, to preserve optimal ventricle filling. 
In practice, during cardiac rehabilitation, a tailored program of exercise should be preceded by a symptom-limited exercise test (cardiopulmonary exercise test if possible). In a patient having a stimulation device, this is a unique opportunity to test the setting of the device.

i) Rate responsiveness

The adaptation of heart rate to the physical activities of the patients as close as possible to the physiologic range can be obtained by the rate responsive function of the PM. Indeed, at exercise, oxygen consumption increase depends on cardiac output, oxygen extraction and delivery to the organs. The increase of cardiac output is usually the major determinant. This is mainly obtained by an increase in heart rate, the increase of stroke volume being a smaller contributor. Numerous parameters and adequate built-in sensors are used to adapt rate response during exercise (activity, QT, temperature, etc.). Although rate response can be obtained in some cases during a cyclo-ergometer test (depending on the type and sensitivity of activity sensors used in the pacemaker), rate response is usually much better during a treadmill test: in patients with pacemaker, the treadmill test should therefore be preferred over the cyclo-ergometer test. Several problems may then be detected: rate responsive function may not have been activated; the upper limit of the PM rate response may induce a fall on the rate slope (i.e., triggering a Luciani Wenchebach or $2 / 1$ phenomenon). The cardiac rehabilitation cardiologist may then ask the electrophysiologist to modify the settings according to the observed abnormalities: adjustment of acceleration slope, upper limit changes, AV delay modifications, etc.

ii) Guarantee of the A-V synchrony

At exercise, the respect of the sequential atrio-ventricular filling allows preserving hemodynamic benefits as compared with a ventricular fixed rate, providing a better cardiac output, a better transport oxygen reserve, and less lactate production. The A-V asynchrony can be observed in different modes of stimulation (i.e., VDD or DDD) and may have a deleterious effect on exercise tolerance, especially in the long term. In most of those cases, the sensibility setting of the atrial lead may be useful; in other cases, the correction of the abnormalities can be more difficult (i.e., over detection of T wave) (Figure 1).

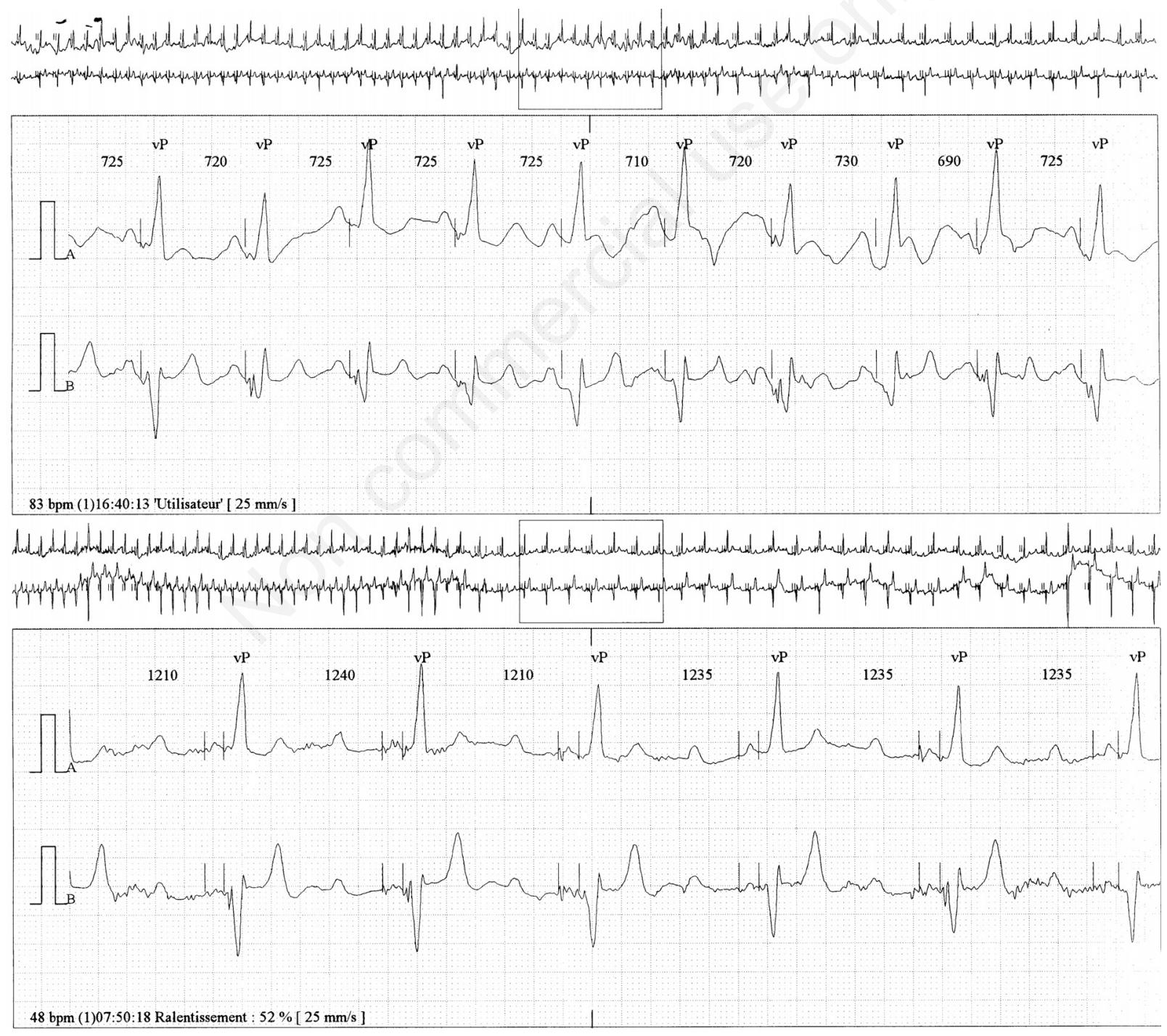

Figure 1.78-year-old woman implanted by a DDD pacemaker. She was complaining of dyspnea at exercise. Holter ECG during an effort shows a sudden slowing down of heart rate with native $\mathrm{P}$ waves not followed by ventricular spikes corresponding to an over detection of $\mathrm{T}$ wave. 
iii) AV delay optimization

The AV-delay plays an important role in the ventricular filling due to the active atrial contraction. This AV-delay may be studied in Doppler echocardiography by analysis of left ventricular filling: a too short AV delay can interrupt the active phase of filling, whereas a too long AV delay may also worsen the filling. However, the setting of PM parameters during an echocardiography is rarely used and reserved to particular cases (such as in heart failure patients). iii) Permanent resynchronization in CRT

CRTs are usually programmed at rest. Assessment during exercise may give important information [7]. Indeed, an exercise test may detect a loss of resynchronization during physical activity that may jeopardize the benefits of CRT (Figure 2). The disappearance of biventricular capture may be caused by a loss of atrial sensing, frequent PVCs, atrial arrhythmias, a spontaneous AV conduction becoming shorter than the programmed AV-delay or to lead movements during exercise.
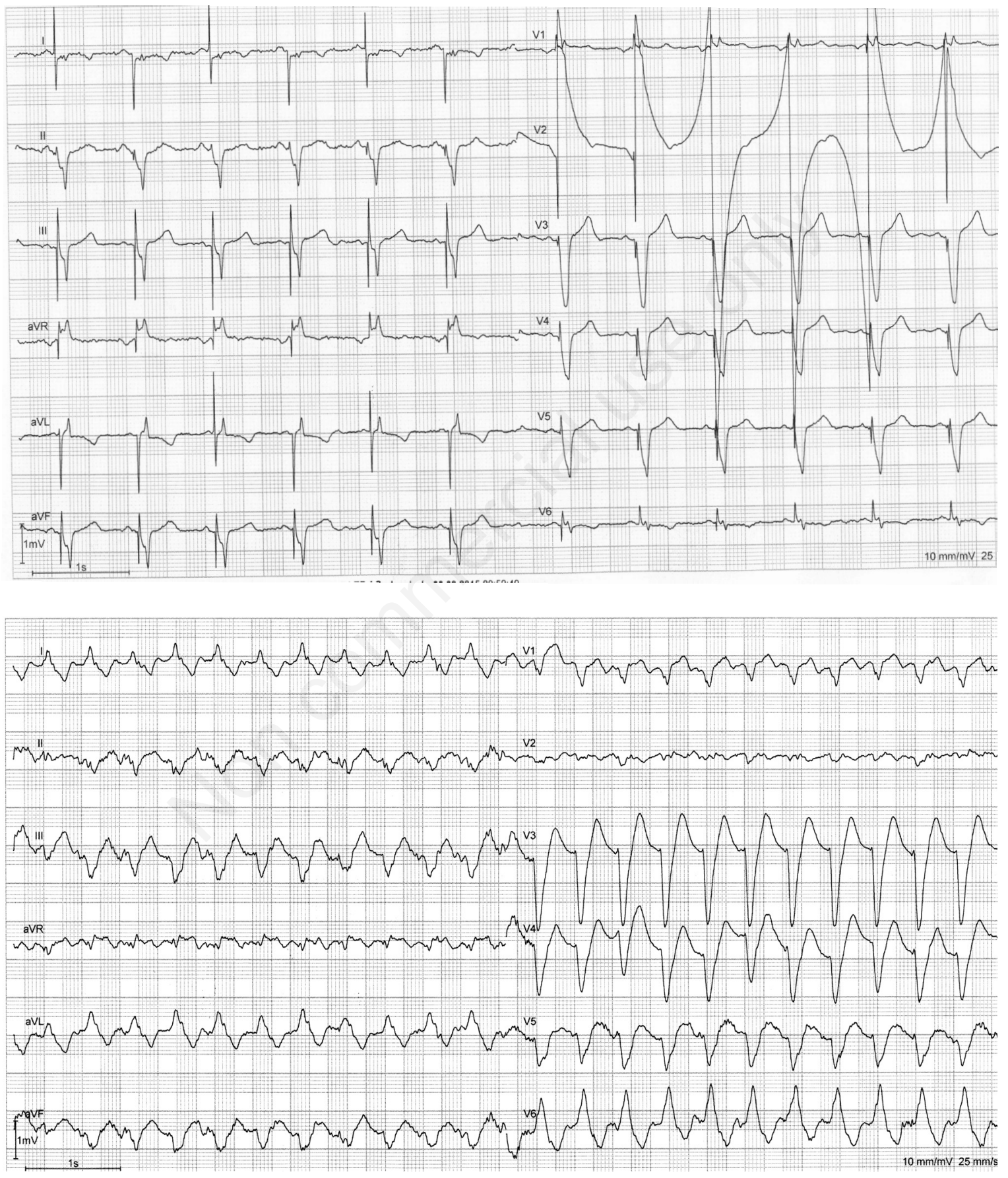

B)

Figure 2. ECG during an exercise echocardiography of a 58-year-old man with CRT for heart failure: A) At rest, HR 75b/min (biventricular capture), and B) at 160 watts workload (loss of biventricular capture). 
Recently, a systematic review analyzed the effects of post-implant optimization of device settings and suggested that the optimization of CRT results in a small but significant improvement in left ventricular function [8]. The benefits of $\mathrm{AV}$ and $\mathrm{VV}$ delays optimization settings are scarcer. Optimization could be used to imiv) ICD prove the exercise performance of a specific patient [9].

Despite the fear of shock occurrence during physical activity, for both patients and physicians, supervised exercise testing is feasible and safe in a medical environment with a well-trained staff. Several types of exercise testing may be proposed [10]. First, the exercise may be limited to a maximum heart rate (HR) 10 to 30 beats below the lowest HR threshold programmed in the device (i.e., below the first anti-tachycardia pacing threshold). This method requires having the programming sheet available. Secondly, exercise may be limited by symptoms. Indeed, these patients are frequently exhausted before the predetermined heart rate threshold; they most frequently receive drugs with negative chronotropic properties that limit their maximum heart rate. Finally, in some rare cases, the ICD may be temporarily switched off during the test, but this way is not generally used in cardiac rehabilitation.

\section{Cardiac rehabilitation in patients implanted with electrophysiological devices}

\section{Cardiac resynchronization therapy}

\section{CRT effects on exercise}

The first description of biventricular pacing as a treatment of advanced heart failure patients, was followed by some randomized studies that showed clinically relevant improvements, among which the exercise capacity. MUSTIC, PATH-CHF, MIRACLE, MIRACLE-ICD, CONTAK ICD, COMPANION and CARE-HF trials have demonstrated improvements in functional class, quality of life, left ventricular ejection fraction and remodeling, and for the two last a benefit in terms of hospitalizations and mortality. In the current guidelines patients with NYHA class II-III despite optimized medical treatment, left ventricular ejection fraction less than $35 \%$, in sinus rhythm and with a QRS duration >120 msec are considered to receive a CRT (class I, level of evidence A) [11].

Exercise tolerance, as measured by a 6 -min walk test and $\mathrm{VO}_{2}$ peak, was modestly but significantly improved (Table 1). These observed improvements are mainly due to an improvement in the haemodynamic profile and to a sympatho-inhibitory effect, leading to reversal skeletal myopathy and, consequently, enhanced exercise performance. Indeed, cardiac resynchronization causes an increase in LVEF and contractility and a reduction in functional mitral regurgitation, resulting in in- creased cardiac output and dp/dt index. These improvements in cardiac haemodynamics then result in a decrease in muscle sympathetic nerve activity, reversal muscle inflammation and improve on the long-term skeletal myopathy.

Moreover, the most substantial improvements in exercise capacity are obtained in patients with the lower baseline peak $\mathrm{VO}_{2}$ [12].

In patients with CRT, exercise capacity response is also determined by the underlying rhythm and heart rate. In summary, a lower mean heart rate is associated with better clinical and hemodynamic response to CRT. In atrial fibrillation patients, one study suggests that beneficial effects are more pronounced after AV node ablation [13].

Lastly, the optimization of CRT has led to a small but significant improvement in LVEF with a non-significant improvement in the 6-min walk test [8]. A recent study, which evaluated the A-V and V-V delays optimization, has shown that there was no consistent pattern of change in gas exchange measures across subjects, and consequently only the optimization of $\mathrm{AV}$ and $\mathrm{VV}$ timing delays on an individual basis may improve the gas exchange $\left(\mathrm{VE} / \mathrm{VCO}_{2}\right.$, oxygen pulse and $\left.\mathrm{PETCO}_{2}\right)$ measures [14].

\section{Cardiac rehabilitation in CRT patients}

Cardiac rehabilitation is a widely accepted management for chronic heart failure patients, particularly those who have been implanted with a CRT device. However, cardiac rehabilitation should be seen as a comprehensive global management. In this way, medical treatment adjustments, patient education, nutrition counseling, psychosocial support and exercise training all contribute to the benefits of cardiac rehabilitation. As a matter of fact, some guidelines include a specific indication of cardiac rehabilitation for the CRT patients [3]. As both endurance training and CRT improve exercise tolerance in heart failure patients, the combination of the two types of management enhances the benefits. So, after a CRT implantation, referral to a cardiac rehabilitation program improves exercise capacity, hemodynamic status, quality of life, quality of sleep, and reduces depression and endothelial dysfunction [15-18].

CRT device improve the central cardiac function but without major peripheral skeletal muscle changes, except muscle sympathetic nerve activity [19]. Conversely, appropriate and personalized exercise training brings further significant improvements in exercise duration, peak $\mathrm{VO}_{2}$, peak of cardiac power output and cardiac reserve, with a significantly better skeletal muscle function depending on the frequency and duration of the exercise period [18]. So, in these patients, the clinical improvements observed in NHYA functional class and quality of life are likely to be explained by both the enhancement of cardiac function induced by the device and by the improved peripheral (muscular and vascular) and cardiac effects of exercise.

All exercise training program in these patients should include, as for all cardiac rehabilitation programs, at least an initial evaluation

Table 1. Improvement on exercise capacity related with CRT placement.

\begin{tabular}{|c|c|c|c|c|}
\hline Trial & Year & n. & $\begin{array}{c}\text { Gain on 6MWD } \\
\text { Control vs CRT (m) }\end{array}$ & $\begin{array}{c}\text { Gain on peak VO } \\
\text { Control vs CRT (mL/kg/min) }\end{array}$ \\
\hline Mustic & 2001 & 58 & -24 us 49 & 1.2 vs 1.7 \\
\hline Path-CHF & 2002 & 53 & 44 vs 47 & 1.8 vs 2.5 \\
\hline Miracle & 2002 & 453 & 10 vs 29 & 0.2 vs 1.1 \\
\hline Miracle-ICD & 2003 & 636 & 52 vs 55 & 0.1 vs 1.1 \\
\hline Contak-CD & 2000 & 490 & 15 vs 35 & 1.2 vs 2.4 \\
\hline Companion & 2008 & 1520 & 1 vs 40 & - \\
\hline
\end{tabular}

6MWD, 6-min walking distance; $\mathrm{m}$, meters; CRT, cardiac resynchronization. 
by cardiopulmonary exercise test and cardiac function evaluation. The personalized exercise prescription may include both endurance and resistance training. Endurance training may use continuous and/or interval or intermittent training models, 3 to 5 days/week, during 30 to $60 \mathrm{~min}$, associated with dynamic exercises. Resistance training sessions ( 2 to 3 sessions/week) may be tailored according to a preliminary evaluation of strength, but requires special attention in order to avoid important strain in the early phase after device placement. Post-hoc analyses of HF-Action, the largest randomized trial of exercise in heart failure patients, have been conducted in patients with a right ventricular or biventricular pacemaker. In this analysis, 435 patients had CRT: 224 were allocated in the exercise training group and 211 in the usual care group. Patients in the exercise training group improved their $\mathrm{VO}_{2}$ peak from $13.8 \mathrm{~mL} / \mathrm{kg} / \mathrm{min}$ to $14.9 \mathrm{~mL} / \mathrm{kg} / \mathrm{min}$ while in the usual care group, $\mathrm{VO}_{2}$ peak remained constant at $13.9 \mathrm{~mL} / \mathrm{kg} / \mathrm{min}$, However, all-cause or CV death or hospitalization for heart failure were not statistically different, with an overlap of confidence interval in this particular group $(p=0.72)$ [20]. Moreover, the other components of cardiac rehabilitation, such as patient education, treatment adjustments, close monitoring; life style recommendations and psychosocial support also have a crucial role in patient outcomes.

Finally, as these devices are now increasingly used, the tele-monitoring of devices coupled with guided home-based training may be proposed to these patients, alone or after an initial center-based cardiac rehabilitation program in order to improve the long-term adherence. To date, too few data are currently available for this kind of programs [21]. Further studies are required.

\section{Implantable cardioverter defibrillator}

\section{Cardiac rehabilitation and ICD}

For several decades, patients with malignant ventricular arrhythmias have derived strong benefit from an ICD implantation. Until recently, these patients have been seldomly referred for exercise training and cardiac rehabilitation in fear of inappropriate shock delivery during exercise and ignorance about the benefit and safety of cardiac rehabilitation. However, in the last 10 years, some studies [22,24,2732 ] have demonstrated the feasibility, safety and beneficial effects of cardiac rehabilitation in these patients (Table 2).

Beneficial effects of training exercise in these patients with ICD have been observed by comparison with the other cardiac patients and with regards to specific psychological aspects. Exercise training in patients with ICD improves significantly the exercise capacity, as much as in the other cardiac patients. Patients with ICD should be referred to cardiac rehabilitation according to the guidelines, not only for the underlying cardiac disease indications, but also after an ICD implantation per se in primary arrhythmogenic cardiopathies. Indeed, among 9 studies, 8 found significant improvements by 15 to $30 \%$ in exercise capacity (Table 2). Different protocols of exercise training have been used, including aerobic high intensity interval training with 4 -min intervals at $85 \%$ of maximal heart rate interrupted by 3 -minute active recovery at $60-70 \%$ of maximal heart rate [22]. Besides a strong gain in exercise capacity, results showed a significant improvement in endothelial function. Interestingly, the effects of exercise training were highly dependent on adherence to the high-intensity levels. Center-based cardiac rehabilitation, with professional supervision and individualized monitoring, offers a unique opportunity for these patients to improve their exercise capacity on the safe side.

The multidisciplinary approach in cardiac rehabilitation also include management of the primary cardiac disease (i.e., ischemic cardiac disease, heart failure), of the medical treatments, cardiovascular risk factors and emphases return to work.

\section{Arrhythmias and safety issues}

It is well known that the risk of arrhythmias is increased by acute exercise. This higher risk is thought to be attributed to adrenergic stimulation, myocardial ischemia, increase in $\mathrm{MVO}_{2}$, decrease in ventricular fibrillation (VF) threshold, or to some specific underlying (and sometimes previously unrevealed) cardiac diseases such as long QT syndrome, hypertrophic cardiomyopathy, arrythmogenic right ventricular cardiomyopathy or cathecholaminergic polymorphic ventricular tachycardia. Both ventricular or supraventricular arrhythmias may be triggered by exercise; however, only ventricular arrhythmias need a shock, namely appropriate shock, whereas a shock delivered by a cardioverter during a supraventricular arrhythmia is inappropriate.

Nevertheless, the risks of severe ventricular arrhythmias (VT of VF) during exercise testing depend on the underlying cardiac disease; and on the previous history of VT/NF (2.3\% of VT/NF if previous history $v s$ $0.05 \%$ if not).

Malignant arrhythmias that may occur during exercise training will be appropriately shocked. But, the occurrence of a supraventricular arrhythmia may trigger an inappropriate shock by the cardioverter, and should therefore be prevented. In order to prevent inappropriate shocks, the following measures are recommended: stop the exercise testing and training 10-20 beats/min below the programmed zones of therapies, continuously monitor these patients during exercise training, use appropriate beta blocker treatment and monitor its effect on max HR, detect and reprogram ICD in case of malfunction, prior to the beginning of the rehabilitation program.

Table 2. Results of exercise training in ICD patients.

\begin{tabular}{|c|c|c|c|}
\hline Trial & n. & Gain & Shocks \\
\hline Fitchet (2003) [27] & 16 ICD pts ExT (no control) & Ex duration $+16 \%$ & 0 \\
\hline Vanhees (2004) [28] & 92 ICD ExT us control (no ICD) & $18 \%$ vs $27 \%$ & $\begin{array}{l}11 \text { appropriate } \\
1 \text { inappropriate }\end{array}$ \\
\hline Davids (2005) [29] & 28 ICD ExT us 54 ICD control & 21.5 vs $14.5 \mathrm{MET} /$ week & appropriate $14 \%$ vs $28 \%$ \\
\hline Fan (2009) [30] & 42 ICD Ex T vs 42 control (no ICD) & $30 \%$ us $37 \%$ & 1 shock vs 0 \\
\hline Smialek (2013) [31] & 45 ICD ExT pts (no control) & $14 \%$ & - \\
\hline Piccini (2013) [32] & 546 ICD ExT vs 507 ICD control & $4.8 \%$ vs $0.8 \%$ & $20 \%$ vs $22 \%$ \\
\hline Isaksen (2015) [22] & 38 ICD ExT us 12 ICD controls & $5.7 \%$ us $4.1 \%$ & 1 shock during Ex \\
\hline Berg S (2015) [24] & 99 ICD ExT us 94 ICD control & $12 \%$ vs $3 \%$ & 0.20 shock $/$ pt $v$ s $0.43 \mathrm{~s} / \mathrm{pt}$ \\
\hline
\end{tabular}


In a review of literature, Isaksen et al. [23] identified ten ICD therapies ( 7 shocks) related to exercise in 834 patients who performed an average of 9.6 weeks of exercise training [incidence $=8 / 1000$ patientstraining-weeks]; in 3 studies he also found that sedentary patients (not involved in a rehabilitation program) experienced more ICD discharges than patients undergoing exercise training. In the 1053 patients with an ICD included in HF Action, results shown that, over the 2 years of follow-up, $20 \%$ of patients in the exercise group had a shock versus $22 \%$ in the control group. More generally, in all reported studies including ICD (alone or with CRT) patients comparing exercise training versus usual care, exercise training was not associated with an increasing occurrence of shocks; arrhythmic events were perhaps even reduced in the long term. Trivially, phantom shocks experienced by $10 \%$ of the ICD patients in the COPE-ICD trial seem not to be influenced by age, sex nor exercise training and psycho-education intervention [24]. Despite safety signals in the medical literature, ICD patients still have a low referral rates and poor adherence in cardiac rehabilitation. This observation might be attributed to the fear of ICD discharges as well as to the high incidence of anxiety and depression in these patients, occurring in $18-38 \%$ and 28 to $32 \%$, respectively [25].

A comprehensive cardiac rehabilitation nowadays includes not only exercise training, which can play in itself a positive role in reassurance in daily living activities, but also psycho-educational support that is particularly important in ICD patients. Improvements on quality of life, positive effects on different scales of anxiety, depression, well-being, general functioning are described using different interventions as cognitive-behavioral, relaxation, personalized or group therapies. The results of the recent COPE-ICD study are consistent with the previous studies with a significant improvement at 3,6 and 12 months, with more pronounced positive effects found only in men [26].

\section{Conclusions}

Cardiac rehabilitation for patients, most of them with heart failure, with implanted electronic devices as pacemakers or ICD is a unique opportunity to optimize the medical treatment, increase their exercise capacity, improve their clinical condition and supervise the functioning of the device. The peripheral effects of exercise training maximize the central benefits offered by the cardiac implanted devices.

\section{References}

1. Piepoli MF, Corrà U, Adamopoulos $\mathrm{S}$, et al. Secondary prevention in the clinical management of patients with cardiovascular diseases. Eur J Prev Cardiol 2014;21:664-81.

2. Fletcher GF, Ades PA, Kligfield P, et al. Exercise standards for testing and training: a scientific statement from the American Heart Association. Circulation 2013;128:873-934.

3. Pavy B, Iliou MC, Vergès Patois B, et al. French society of cardiology guidelines for cardiac rehabilitation in adults. Arch Cardiovasc Dis 2012;105:309-28.

4. Mond HG, Proclemer A. The $11^{\text {th }}$ world survey of cardiac pacing and implantable cardioverter-defibrillators: calendar 2009 - a World Society of Arrhythmia's project. Pacing Clin Electrophysiol 2011;34:1013-27.

5. Flynn KE, Piña IL, Whellan DJ, et al. Effects of exercise training on health status in patients with chronic heart failure: HF-ACTION randomized controlled trial. JAMA 2009;301:1451-9.
6. Mc Murray J, Packer M, Desai A, et al. Angiotensin-neprilysin inhibition versus enalapril in heart failure. N Engl J Med 2014; 371:993-1004.

7. Bax JJ, Abraham T, Barold SS, et al. Cardiac resynchronization therapy. J Am Coll Cardiol 2005;46:2153-82.

8. Kosmala W, Marwick T. Meta-analysis of effects of optimization of cardiac resynchronization therapy on left ventricular function, exercise capacity, and quality of life in patients with heart failure. Am J Cardiol 2014;113:988-94.

9. Kim CH, Cha YM, Shen WK, et al. Effects of atrioventricular and interventricular delay on gas exchange during exercise in patients with heart failure. J Heart Lung Transplant 2014;33:397-403.

10. Qian Z, Guo J, Zhang A, et al. Optimal programming management of ventricular tachycardia storm in ICD patients. J Biomed Res 2015;29:35-43.

11. European Society of Cardiology (ESC), European Heart Rhythm Association (EHRA), Brignole M, et al. 2013 ESC Guidelines on cardiac pacing and cardiac resynchronization therapy: the task force on cardiac pacing and resynchronization therapy of the European Society of Cardiology (ESC). Europace 2013;15:1070-111.

12. Arora S, Aarones M, Aakhus S, et al. Peak oxygen uptake during cardiopulmonary exercise testing determines response to cardiac resynchronization therapy. J Cardiol 2012;60:228-35.

13. Gasparini M, Auricchio A, Regoli F, et al. Four-year efficacy of cardiac resynchronization therapy on exercise tolerance and disease progression. J Am Coll Cardiol 2006;48:734-43.

14. Kim CH, Cha YM, Shen WK, et al. Effects of atrioventricular and interventricular delays on gas exchange during exercise in patients with heart failure. J Heart Lung Transplant 2014;33:397-403.

15. Iliou MC., Alonso C, Cristofini P, et al. Exercise training after cardiac resynchronization in chronic heart failure. Results of a pilot study. Eur J Cardiovasc Prev Rehab 2004;10:402.

16. Belardinelli R, Capestro F, Misiani A, et al. Moderate exercise training improves functional capacity, quality of life, and endothelium-dependent vasodilation in chronic heart failure patients with implantable cardioverter defibrillators and cardiac resynchronization therapy. Eur J Cardiovasc Prev Rehabil 2006; 13:818-25.

17. Conraads V, Vanderheyden M, Paelinck B, et al. The effect of endurance training on exercise capacity following cardiac resynchronization therapy in chronic heart failure patients: a pilot trial. Eur J Cardiovasc Prev Rehabil 2007;14:99-106.

18. Patwala A, Woods P, Sharp L, et al. Maximizing patient benefit from cardiac resynchronization therapy with the addition of structured exercise training. J Am Coll Cardiol 2009;53:2332-9-

19. Kuniyoshi RR, Martinelli M, Negrao CE, et al. Effects of cardiac resynchronization therapy on muscle sympathetic nerve activity. Pacing Clin Electrophysiol 2014 37:11-8.

20. Zeiltler E, Piccini J, Hellkamp A et al. Exercise training and pacing status in patients with heart failure: results from HF-ACTION. J Cardiac Fail 2015;21:60-7.

21. Smolis-Bak E, Dabrowski R, Piotrowicz E, et al. Hospital-based and telemonitoring guided home-based training programs: effects on exercise tolerance and quality of life in patients with heart failure (NYHA class III) and cardiac resynchronization therapy. A randomized, prospective observation. Int J Cardiol 2015;199:442-7.

22. Isaksen K, Munk P, Valborgland T, et al. Aerobic interval training in patients with heart failure and an implantable cardioverter defibrillator: a controlled study evaluating feasibility and effect. Eur J Prev Cardiol 2015; 22:296-303.

23. Isaksen K, Morken IM, Munk Ps, et al. Exercise training and cardiac rehabilitation in patients with implantable cardioverter defib- 
rillators: a review of current literature focusing on safety, effects of exercise training, and the psychosocial impact of programme participation. Eur J Prev Cardiol 2012;19:804-12.

24. Berg S, Pedersen P, Zwisler AD, et al. Comprehensive cardiac rehabilitation improves outcome for patients with implantable cardioverter defibrillator. Findings from the COPE-ICD randomised clinical trial. Eur J Cardiovasc Nurs 2015;14:34-44.

25. Godermann F, Butter C, Lampe F, et al. Determinants of the quality of life $(\mathrm{QoL})$ in patients with an implantable cardioverter/defibrillator (ICD). Qual Life Res 2004;13:411-6.

26. Berg SK, Elleman-Jenasen L, Zwisler AD, et al. Sexual concerns and practices after ICD implantation: findings of the COPE-ICD rehabilitation trial. Eur J Cardiovasc Nurs 2013;12:468-74.

27. Fitchet A, Doherty PJ, Bundy C, et al. Comprehensive cardiac rehabilitation programme for implantable cardioverter-defibrillator patients: a randomised controlled trial. Heart 2003;89:155-60.
28. Vanhees L, Kornaat M, Defoor J, et al. Effect of exercise training in patients with an implantable cardioverter defibrillator. Eur Heart $\mathrm{J}$ 2004;25:1120-6.

29. Davids J, McPherson C, Early C, et al. Benefits of cardiac rehabilitation in patients with implantable cardioverter-defibrillators: a patient survey. Arch Phys Med Rehabil 2005;86:1924-8.

30. Fan S, Lyon CE, Savage PD, et al. Outcomes and adverse events among patients with implantable cardiac defibrillators in cardiac rehabilitation: a case-controlled study. J Cardiopulm Rehabil Prev 2009;29:40-3.

31. Smialek J, Lelakowski J, Majewski J. Efficacy and safety of early comprehensive cardiac rehabilitation following the implantation of cardioverter-defibrillator. Kardiol Pol 2013;71:1021-8.

32. Piccini J, Hellkamp A, Whellan D, et al. Exercise and implantable cardioverter defibrillator shocks in patients with heart failure: results from HF-ACTION. JACC Heart Fail 2013;1:142-148. 